\title{
Low-Grade Osteosarcoma of the Mandible
}

\author{
Ramen Sinha $\cdot$ S. K. Roy Chowdhury $\cdot$ \\ P. K. Chattopadhyay $\cdot$ K. Rajkumar
}

Received: 8 February 2009/ Accepted: 15 March 2010/Published online: 28 September 2010

(c) Association of Oral and Maxillofacial Surgeons of India 2010

\begin{abstract}
Osteosarcoma (OS), a rare malignant bone tumour arising from primitive bone forming mesenchyme, most often arises in the metaphyses of long bones of the extremities. Bone or osteoid formation within the tumour is characteristic of an osteosarcoma. Craniofacial osteosarcoma (CFOS), most often located in the mandible or maxilla, accounts for only 5-13\% of all osteosarcomas. In general, OS of the jaw is a high-grade lesion. Low-grade lesions are rare and represent less than $2 \%$ of all osteosarcomas reported in the literature. Because of its rarity and well differentiation, Low-grade OS is usually misdiagnosed as a benign lesion. The clinical and radiographic presentation does not correlate well with the subtle histology picture of a low-grade osteosarcoma which makes the diagnosis difficult.
\end{abstract}

Keywords Low-grade osteosarcoma .

Craniofacial osteosarcoma

\section{Introduction}

Osteosarcoma (OS) is a rare malignant bone tumour arising from primitive bone forming mesenchyme; most often arises in the metaphyses of long bones of the extremities. Bone or osteoid formation within the tumour is characteristic of an osteosarcoma. Craniofacial osteosarcoma (CFOS), most often located in the mandible or maxilla, accounts for only $5-13 \%$ [1] of all osteosarcomas and $1 \%$

R. Sinha $(\bowtie)$ · S. K. Roy Chowdhury · P. K. Chattopadhyay · K. Rajkumar

Division of Oral and Maxillofacial Surgery,

Department of Dental Surgery, AFMC, Pune 411040, India

e-mail: ramensden@gmail.com of all head and neck malignancies [2]. Classical osteosarcoma of the long bones most often affects adolescents and young adults. CFOS typically occurs in the third or fourth decade of life [2]. Presenting signs and symptoms of CFOS include regional swelling, pain and parasthesia. The risk of early metastases, particularly in the lung, is high, with $20 \%$ of all patients with osteosarcoma having solid metastases when first diagnosed; the rate of micrometastases is estimated to be $80 \%$ [2]. Head and neck OS are associated with a lower metastatic rate than are long bone OS and they have a better 5-year survival rate, ranging between $10 \%$ and $85 \%$ [3]. Similar to osteosarcoma of the extremities, adequate surgical resection is considered a mainstay of treatment. In general, osteosarcomas of the jaw are highgrade lesions. Low-grade lesions are rare and include the well-differentiated intraosseous osteosarcoma (low-grade central osteosarcoma) and parosteal osteosarcomas. Lowgrade osteosarcoma (LOS; well-differentiated intraosseous osteosarcoma), represents less than $2 \%$ of all osteosarcomas reported in the literature [4]. Because of its rarity and well differentiation, LOS is usually misdiagnosed as a benign lesion.

\section{Case Report}

A 25-year-old female patient reported to this institution with complaints of painless slowly growing swelling of the right side of the lower jaw since past one year. She had initially reported to a private practitioner with complaints of pain and swelling in her lower right posterior tooth for which extraction of her carious lower right molar was done. Though the pain subsided, there was no change in the size of the swelling. Subsequently she was referred to a private hospital where an incisional biopsy of the swelling was 


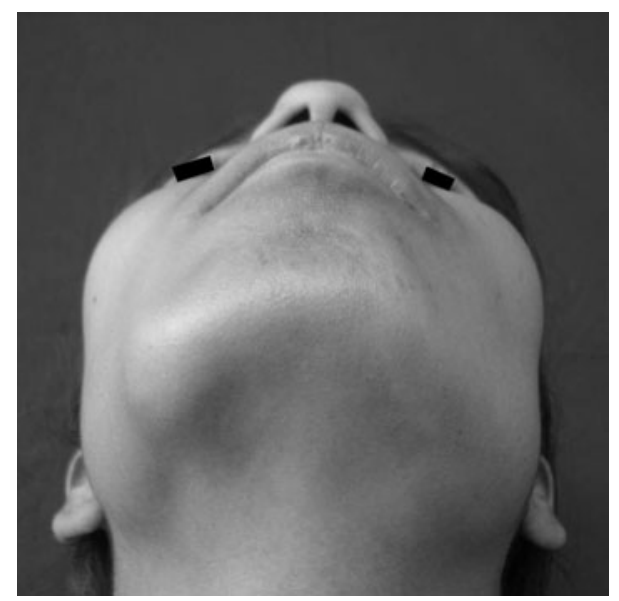

Fig. 1 Extraoral examination showing an ovoid shaped $(6 \times 4 \mathrm{~cm}$ in size) swelling on the right lower border of the mandible

performed and a histopathological diagnosis of Chondromyxoid fibroma was obtained. She then reported to this hospital with the above mentioned complaint.

On clinical examination a painless slow growing ovoid shaped swelling was present on the right body of the mandible approximately $6 \times 4 \mathrm{~cm}$ in size. The swelling extended anteriorly from the commissure of the lip on the right side, and posteriorly to the angle of the mandible. Inferiorly the swelling extended approximately $1 \mathrm{~cm}$ below the lower border of the mandible (Fig. 1). On palpation the swelling was firm, non tender, noncompressible, nonreducible and exhibited no fluctuation or crepitus. There was expansion and thinning of the buccal cortex with parasthesia on the right of the lower lip. A single enlarged, mobile, non tender lymph node was palpable in the right submandibular region. Intraoral examination revealed a painless smooth nodular mass in the lower right alveolar ridge extending from $45-48$ region. The swelling was fixed to the underlying tissues and firm in consistency (Fig. 2). On palpation there was thinning and perforation of the lingual cortex. Based on the clinical findings a provisional diagnosis of fibro osseous lesion of the right body of the mandible was established. Differential diagnosis included central giant cell granuloma, ossifying fibroma, fibrous dysplasia and mesenchymal malignancies of the jaw like osteosarcoma, fibrosarcoma, etc.

\section{Radiologic Findings}

A panoramic radiograph showed a diffuse mixed-density lesion, predominating radiopacity interwoven with radiolucent areas and ill-defined margins involving the right body of the mandible extending from the 44-48 region. There was loss of lamina dura and widened periodontal ligament space of the lower right second premolar and

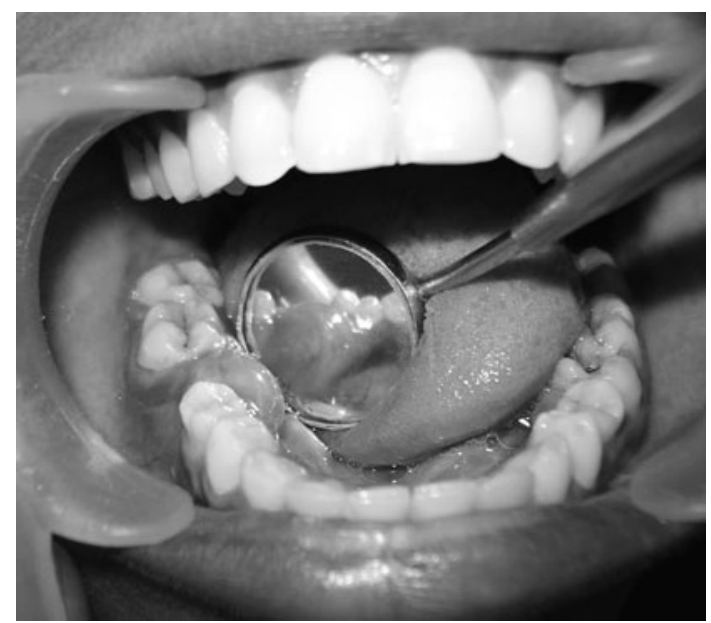

Fig. 2 Intraoral examination showing a smooth nodular mass in the lower right alveolar ridge extending from 45-48 region

second and third molar (Fig. 3). A non enhanced axial and coronal computed tomography showed an expansile lesion within the right hemi-mandible affecting the body of the mandible. The buccal and lingual bone cortices were totally destroyed and the tumour had extended to the soft tissue (Fig. 4). A PA and lateral chest radiograph was obtained to detect the presence of metastatic deposits. No metastasis was found in the conventional postero-anterior and lateral chest radiographs.

\section{Incisional Biopsy}

An incsional biopsy was performed intraorally from the center of the lesion which showed proliferation of small stellate cells and spindle cells in a myxoid matrix. The cells showed moderate nuclear pleomorphism and minimal mitotic activity. A few areas of mineralization, chondroblastic differentiation of the osteoid were also seen suggestive of histopathological picture of osteosarcoma.

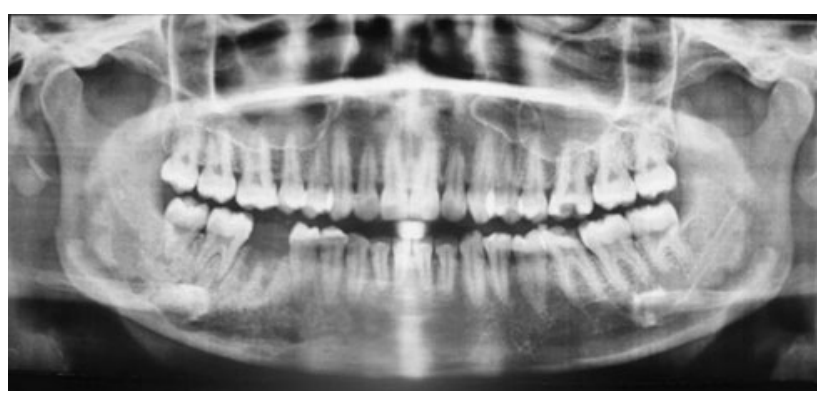

Fig. 3 Panoramic radiograph showing a diffuse mixed-density lesion, predominating radiopacity interwoven with radiolucent areas, and ill-defined margins involving the right mandible body extending from the 44-48 region 


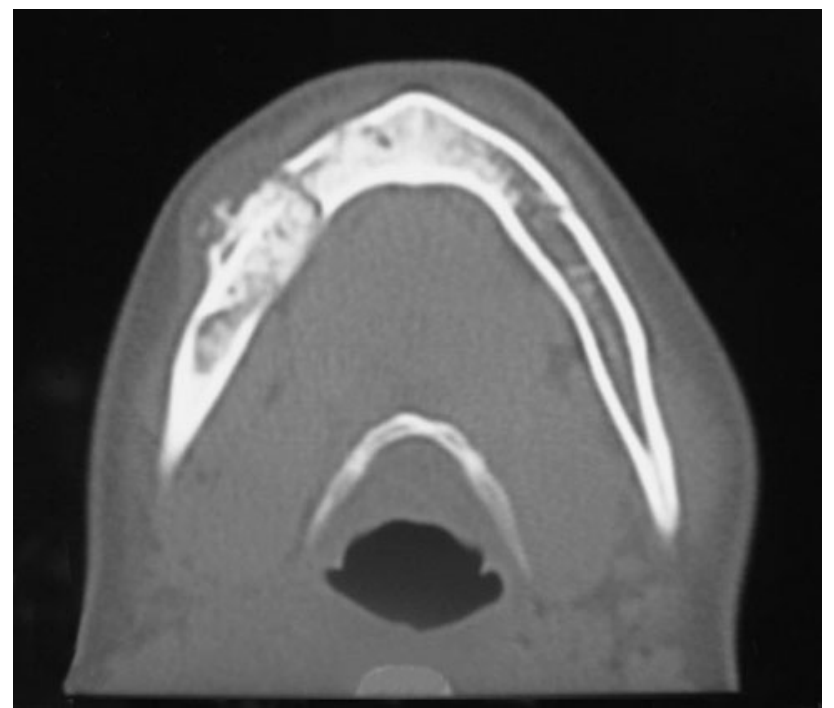

Fig. 4 Axial CT section showing a mixed density lesion of the right body of the mandible with irregular margins, cortical perforation and soft tissue infiltration

\section{Surgical Procedure and Postoperative Follow-Up}

The patient was taken up for surgical resection. Safe bony margins of $2 \mathrm{~cm}$ were maintained from the edges of the tumour during resection (Fig. 5). A solitary enlarged lymph node in the sub-mandibular region was removed. Reconstruction of the defect was done with rigid titanium reconstruction plate. Postoperatively patient was put on an antibiotic course (Inj. Cefotaxime $1 \mathrm{gm}$ i.v bd., Inj. Metronidazole $500 \mathrm{mg} 8 \mathrm{~h}$, Inj. Gentamicin $60 \mathrm{mg}$ i.v $8 \mathrm{~h}$ ) for 5 days and intermaxillary fixation was done with elastics to stabilize the occlusion. Postoperatively patient developed

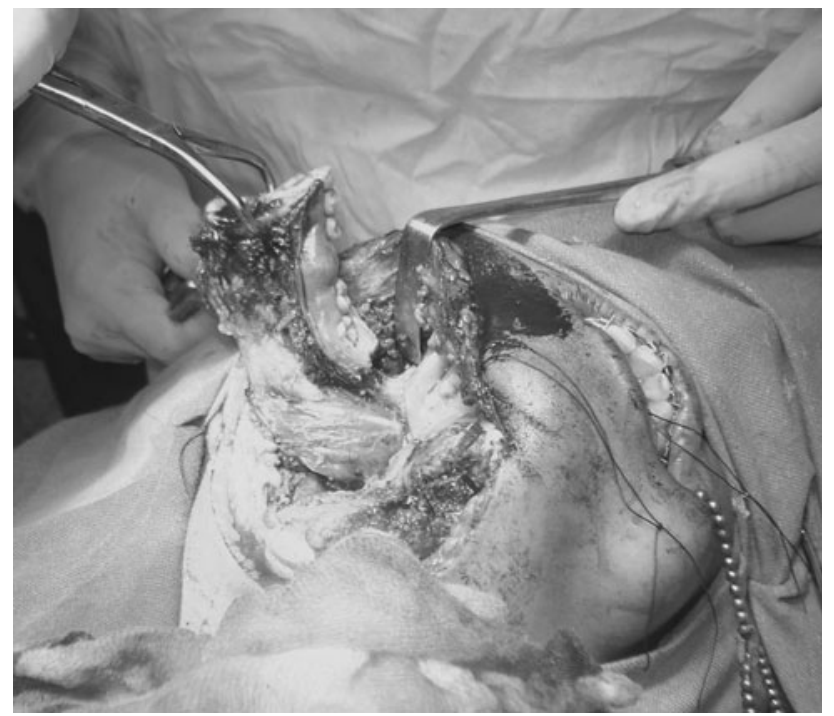

Fig. 5 Surgical resection of the tumour with safe bony margins of $2 \mathrm{~cm}$ wound dehiscence along the anterior edge of the incision with purulent discharge. Surgical exploration was carried out and a surgical drain inserted. Culture and sensitivity testing of the drained pus showed sensitivity for glycopeptide group of antibiotics. Subsequently antibiotic therapy was changed to Inj. Vancomycin $500 \mathrm{mg}$ bd and a second surgical procedure was undertaken to remove the titanium reconstruction plate. The area was approached via the previous incision, reconstruction plate was removed, area debrided thoroughly and primary closure of the surgical wound was done. Postoperatively patient was put on an antibiotic course for 5 days and naso gastric tube feed for 1 week. Subsequent recovery was uneventful and surgical wounds healed well. The individual received Adriamycin and Cisplatin as postoperative chemotherapy. Presently the patient is under regular follow-up and free of disease.

\section{Histopathological Findings}

The surgical specimen consisted of a right hemimandible that measured $8 \times 4 \times 2 \mathrm{~cm}$ in greatest dimension (Fig. 6). The cut surface showed a grey white $3 \times 2 \mathrm{~cm}$ tumour which is firm to hard in consistency destroying the cortical bone and reaching the gingival soft tissue on the superior aspect. No necrosis or haemorrhage was seen in the growth. Multiple representative sections of the tumour showed a variegated appearance with proliferation of small stellate cells and spindle cells in a myxoid matrix. The cells showed moderate nuclear pleomorphism, clumped chromatin and minimal mitotic activity. A few areas of mineralization, chondroblastic differentiation of the osteoid were also seen (Fig. 7). The tumour tissue was seen infiltrating the parent mature woven bone and cortical bone extending into the gingival nodule. Foci of bone destruction were seen in few places with no giant cell

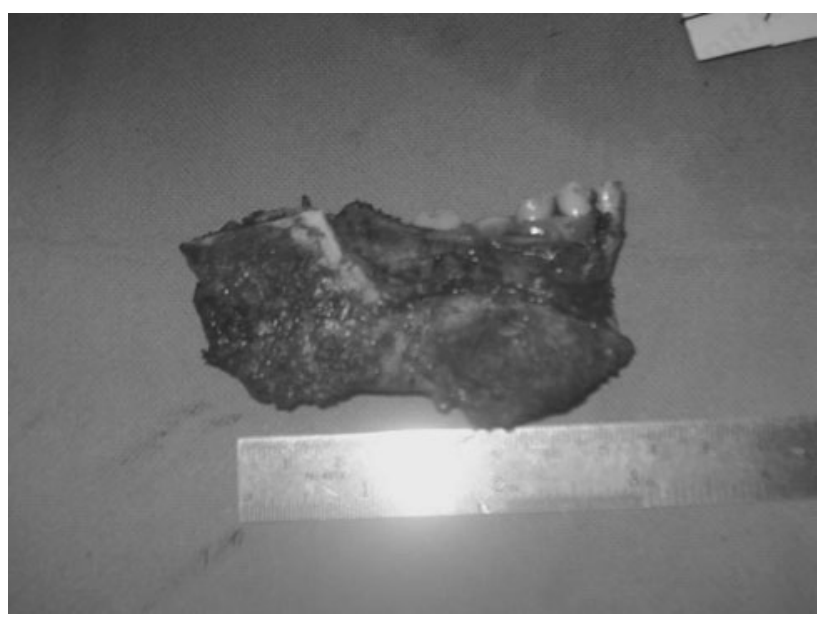

Fig. 6 Resected specimen 


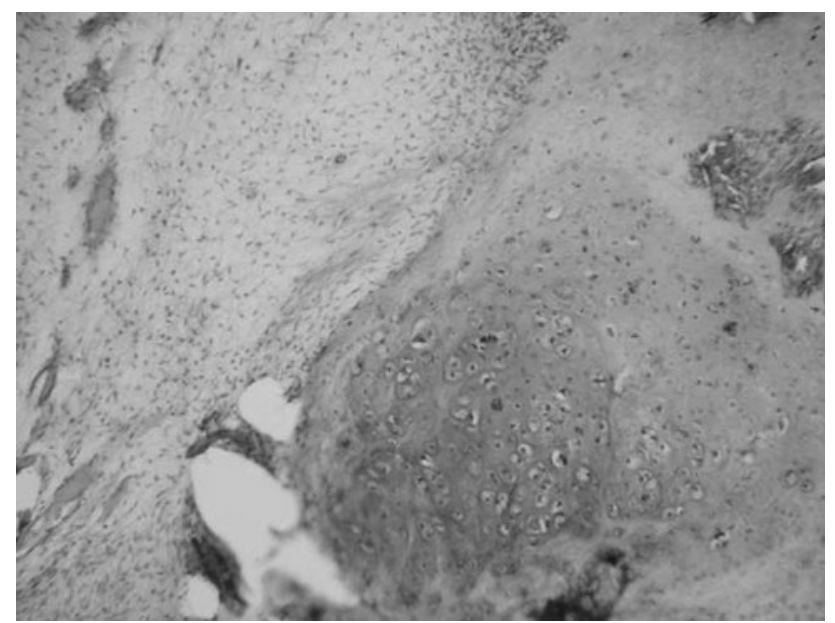

Fig. 7 Photomicrograph of the tumour showing a variegated appearance with proliferation of small stellate cells and spindle cells in a myxoid matrix. The cells showed moderate nuclear pleomorphism, clumped chromatin and minimal mitotic activity. A few areas of mineralization, chondroblastic differentiation of the osteoid can be seen $(H \& E \times 400)$

proliferation. Surgical cut margins were free of tumour by $5 \mathrm{~mm}$. No lymphatic/vascular invasion was seen. A $1.5 \mathrm{~cm}$ lymph node excised during the surgery showed features of reactive follicular hyperplasia. Based on the histopathological findings a grading of stage pT1 N0 Mx low grade G1was reached.

\section{Discussion}

Osteosarcoma of the jaw is a rare entity corresponding to $5-13 \%$ of all cases of skeletal osteosarcomas [1]. Most osteosarcomas of the jaw (87\%) are high-grade lesions, while low-grade lesions are rare and include the well-differentiated intramedullary osteosarcoma (or LOS) and the paraosteal osteosarcoma [4]. Low-grade central osteosarcoma occurs within a broad age range (16-69 years), with peak incidence in the third or fourth decades [5, 6]. Similar to the conventional type of osteosarcoma, LOS has a predilection for the body of the mandible, and usually presents with a long-standing painless swelling [4].

LGCO has variable radiographic appearances and initially it may be mistakenly interpreted as a benign process. Most radiographs and CT scans show a mottled radiopaque or mixed radiolucent-radiopaque appearance in the medullary space. Conditions that may mimic LGCO include fibrous dysplasia, giant cell tumor, nonossifying fibroma, and osteoblastoma [5,7]. The radiologic features in the largest series of LOS reported in the literature are indicative of malignancy in $82 \%$ of cases and include poor margination and cortical destruction with or without extension into soft tissue. The radiologic characteristics of our case showed a diffuse mixed-density lesion; predominating radiopacity interwoven with radiolucent areas; and ill-defined margins involving the right mandibular body.

Common histologic features of LOS in general include a spindle cell proliferation with low cellularity, low mitotic rate (usually less than three mitosis per $10 \mathrm{HPF}$ ), bland or minimal cytologic atypia, and variable osteoid production. Six patterns or subtypes of LOS $[7,8]$ have been described based on the distribution of the osteoid, the amount of collagen, the presence of myxoid change, and the benign lesion it mimics. These include: paraosteal-like, fibrous dysplasialike, desmoplastic fibroma-like, nonossifying fibroma-like, osteoblastoma-like, and chondromyxoid fibroma-like. Our case showed a chondromyxoid pattern with proliferation of small spindle or stellate tumour cells showing minimal mitotic activity with moderate nuclear pleomorphism and hyperchromatism, set in a highly myxoid stroma superficially mimicking chondromyxoid fibroma. A few areas of mineralization, chondroblastic differentiation of the osteoid were also seen. The histological hallmark was the direct production of osteoid by tumour cells. Chondromyxoid fibroma-like osteosarcoma merits recognition as a distinct variant of low-grade osteosarcoma for which early appropriate surgery is indicated [9].

Accurate diagnosis of LOS is based on the aggressive behaviour of the lesion (poor margination, cortical bone destruction, and invasion of soft tissue) seen in the radiograph and not in the histologic characteristics of the lesion (cellularity, atypia, mitosis) [8]. Therefore, interpretation of small biopsies is very difficult, unless there is radiologic evidence of a destructive lesion. In our case the biopsy was interpreted as a spindle cell proliferation of low malignant potential.

The differential diagnosis of LOS includes benign and low-grade spindle lesions such as fibrous dysplasia, ossifying fibroma, chondromyxoid fibroma, unclassifiable fibro-osseous lesions, as well as desmoplastic fibroma. Of these lesions, the most difficult to distinguish from LOS is fibrous dysplasia [6,7]. What makes fibrous dysplasia so difficult to distinguish from LOS in the jaw is that unlike the long bones, fibrous dysplasia of the jaws tends to be diffuse [6]. Also similar to LOS, fibrous dysplasia is associated with trabecular bone, but the presence of an infiltrative pattern (rare in fibrous dysplasia), subtle cytologic atypia and the atypical roentgenographic features allow the distinction between these two entities. The invasive growth pattern is the most helpful feature in differentiating LOS from fibrous dysplasia.

Resection with wide surgical margins is the most important factor for prognosis and provides a 5-year survival rate of $80 \%$ [1]. Bony margins for resection should at least be $2 \mathrm{~cm}$ from the clinical-radiographic edge or the nearest suture in the midface. Soft tissue margins around an osteosarcoma resection should be $2 \mathrm{~cm}$ or more and 
assessed with frozen sections. Adjuvant chemotherapy or radiotherapy seems to be inefficacious in cases of LGCO [7]. Recurrences of LGCO may exhibit a higher histopathological grade or dedifferentiation with potential for metastases that can lead to the death of the patient [10]. The transformation of a low-grade central osteosarcoma into a high-grade osteosarcoma on recurrence has been reported in $15 \%$ of the patients [7].

Low-grade central osteosarcoma is a rare neoplasm that histopathologically offers the potential for misdiagnosis as benign condition, most specifically fibrous dysplasia. Careful attention to clinical, radiographic, and microscopic findings is necessary to ensure establishment of the correct diagnosis, thus helping to prevent incorrect treatment and unfortunate outcome. Prognosis is good if an early diagnosis of the lesion is made with prompt surgical intervention.

In summary, we have presented an unusual case of LOS of the jaw in a 25-year-old woman. Cortical destruction or invasion of soft tissue is the most important criteria for diagnosis. Such tumour should be considered in the differential diagnosis of lesions of the jaw, taking into account that the radiologic studies cannot always be interpreted as malignant.

\section{References}

1. Mardinger O, Givol N, Talmi YP, Taicher S (2001) Osteosarcoma of the jaw. The Chaim Sheba Medical Center experience.
Oral Surg Oral Med Oral Pathol Oral Radiol Endod 91(4): 445-451

2. Thiele OC, Freier K, Bacon C, Egerer G, Hofele CM (2008) Interdisciplinary combined treatment of craniofacial osteosarcoma with neoadjuvant and adjuvant chemotherapy and excision of the tumour: a retrospective study. Br J Oral Maxillofac Surg 46(7):533-536

3. Forteza G, Colmenero B, López-Barea F (1986) Osteogenic sarcoma of the maxilla and mandible. Oral Surg Oral Med Oral Pathol 62(2):179-184

4. Diniz AF, Filho JA, Alencar Rde C, Garcia RR, Silva MR, Ribeiro-Rotta RF, Silva MA, Batista AC, Mendonça EF (2007) Low-grade central osteosarcoma of the mandible: a case study report. Oral Surg Oral Med Oral Pathol Oral Radiol Endod 103(2):246-252

5. Zhao W, Cure J, Castro CY (2002) Low-grade osteosarcoma of the jaw. Ann Diagn Pathol 6(6):373-377

6. Bertoni F, Bacchini P, Fabbri N, Mercuri M, Picci P, Ruggieri P, Campanacci M (1993) Osteosarcoma. Low-grade intraosseoustype osteosarcoma, histologically resembling parosteal osteosarcoma, fibrous dysplasia, and desmoplastic fibroma. Cancer 71(2):338-345

7. Kurt AM, Unni KK, McLeod RA, Pritchard DJ (1990) Low-grade intraosseous osteosarcoma. Cancer 65(6):1418-1428

8. Raubenheimer EJ, Noffke CE (1998) Low-grade intraosseous osteosarcoma of the jaws. Oral Surg Oral Med Oral Pathol Oral Radiol Endod 86(1):82-85

9. Chow LT, Lin J, Yip KM, Kumta SM, Ahuja AT, King WW, Lee JC (1996) Chondromyxoid fibroma-like osteosarcoma: a distinct variant of low-grade osteosarcoma. Histopathology 29(5): 429-436

10. Bennett JH, Thomas G, Evans AW, Speight PM (2000) Osteosarcoma of the jaws: a 30-year retrospective review. Oral Surg Oral Med Oral Pathol Oral Radiol Endod 90(3):323-332 